\title{
Numerical simulation of the unsteady flow field in the human pulmonary acinus
}

\author{
DOGAN CILOGLUD
}

\author{
Ataturk University, Vocational College of Technical Sciences, Erzurum, Turkey \\ e-mail: dciloglu@atauni.edu.tr
}

MS received 11 January 2021; revised 12 May 2021; accepted 5 August 2021

\begin{abstract}
Understanding of airflow dynamics in the human pulmonary acinus is important for increasing targeted drug effectiveness and determining the health impact of toxic aerosols. However, there is a lack of quantitative data about the pulmonary airflow in realistic and flexible idealized geometries. This paper aims to numerically analyse the flow field of the pulmonary acinus using the computational fluid dynamics (CFD) model during transient breathing. Three-dimensional models with rhythmically expanding-contracting alveolar walls were developed for representing the pulmonary region of the human lung. Three different breathing scenarios were applied in the CFD simulations. The results showed that the transient flow conditions determined the transitions between flow types. The recirculating flow in the alveoli was observed for all cases and it was determined that its intensity depended on the breathing scenario. The flow velocity in the alveoli was slower than that of the channel flow. As we moved deeper into the lung, the flow pattern inside the alveoli exhibited a radial velocity profile. It was found that the alveolar flow exhibited a typical stenotic channel flow characteristics. As a result, the acinus models used in this study takes into account the alveolar wall motion based on physiological breathing conditions. To simulate or estimate the airflow dynamics, thus, the results obtained in this study can be easily utilized in the human lung airway models.
\end{abstract}

Keywords. Acinar flow dynamics; CFD modeling; breathing conditions; wall motion; Covid-19.

\section{Introduction}

The new type of coronavirus disease (Covid-19) is a new viral respiratory disease that has been firstly identified with high fever and shortness of breath in Wuhan, China, on January 13, 2020. Covid-19 has become a pandemic, mainly transmitted from person to person through close contact and droplets, with respiratory symptoms such as coughing or sneezing of an infected person. The droplets inhaled into the lungs lead to respiratory disorders such as pneumonia and acute respiratory distress syndrome (ARDS). While most Covid-19 deaths occur in older people and especially those with chronic diseases, even young people may experience serious complications. Moreover, the lasting damage can occur in people who survive the disease, known as post-Covid fibrosis. In this case, the fluid fills in the small air sacs called alveoli in the lungs, and thus ARDS occurs. This reduces oxygen in the bloodstream and leads to organ failure. Currently, there is no fully documented treatment modality for the treatment of post-inflammatory pulmonary fibrosis after Covid-19 infection. However, subsequent studies have found that some treatments such as steroids [1, 2], spironolactone [3-5], fibrinolytic agents [6-9], and antiviral agents [10-13] are promising in preventing the post-Covid fibrosis. Covid-19 can also cause the immune system to form blood clots, which prevents blood from going to certain parts of the lung. As a result, the holes form in the lungs and the lungs die. The aerosol therapy is a basic and common method used to treat pulmonary diseases at home and healthcare facilities. In these days when the treatment of respiratory diseases becomes important, it is vital to accurately determine the airflow dynamics in the human pulmonary acinus for efficient treatment methods.

Our lungs are the most important and the largest part of the human respiratory system. Initially starting with the left and right main bronchi, and then separating from numerous bronchus and bronchioles, the structure of the human lung airways eventually forms a tree view. Starting from generation 0 , there are a total of 23 generations in the human lungs of which the first 16 belong to the well-known tracheobronchial region and the remaining ones belong to the alveolated ducts [14]. In a healthy adult, there are over 500 million alveoli [15], where gas exchange occurs, i.e., oxygen input to the blood and carbon dioxide output from the blood. The gas exchange zone with alveolated ducts and sacs is known as the alveolar or acinar region. This region is a crucial zone in which the transmission of toxic particles 
should be prevented and therapeutic drug particles should be effective.

In the last decade, several studies have been carried out on the flow and aerosol particle behavior in the human lung airways using in vivo, in vitro, and in silico approaches. It is so difficult to obtain meaningful in vivo data due to accuracy and safety concerns related to human and animal subjects [16-18]. In vitro studies have been especially performed in the upper respiratory and the conducting airways [19-23], while in silico studies have provided the results in the pulmonary region of the human lung [24-38]. In a numerical method, by employing CFD on real or idealized model geometries, accurate predictions for fluid and particle transport in the alveolar region have been obtained. The typical process for such studies begins with determining a realistic geometry, followed by the use of a specialized software program on which the governing equations can be solved.

Hofmann et al [24] used an asymmetric bifurcation airway model representing the generations 15-16 for low and heavy physical activity breathing conditions. Darquenne [25] investigated the flow field in the alveolar region of the human lung on a more complex 2D multi-generation structure of alveolated ducts. Harrington et al [26] developed the first 3D bifurcation model of a fully alveolated ducts representing generations $18-22$ with rectangular alveoli compartments. Ertbruggen et al [27] compared the numerical predictions and experimental results of the acinar flow in a 3D alveolated bend geometry with rigid walls. Darquenne et al [28] used the first 3D model of fully alveolated duct with moving walls representing the generations 18-23. They simulated the transport and deposition of the particles ranging from 1 to $5 \mu \mathrm{m}$ in diameter in the acinar region of the lung. Kumar et al [29] simulated the flow analysis in the acinar region using a 3D honeycomblike polygonal model. Their numerical results showed that the flow into and out of the alveoli was formed by the alveolar wall motion and it was characterized by the flow entrainment regions. In another study by the same authors [30], they reported that acinar flow belongs to a unique flow category with low Reynolds numbers and high oscillations.

Salma et al [31] examined the effect of physical exertion on aerosol deposition in the whole respiratory system. They reported that the level of physical exertion during a single breath plays a significant role in the fraction of particle deposition. Kolanjiyil and Kleinstreuer [32] employed a new whole-lung-airway model (WLAM) to simulate the airflow and particle behavior for different inhalation/exhalation scenarios (normal, light, and heavy activity). The authors suggested the use of their whole-lung model for analyzing human exposure to toxic particulate matter or estimating the pharmacological effects of administered drug-aerosols. However, their models did not take into account the alveolar wall motion. Ma and Darquenne [33] simulated the airflow and depositions of 1 and $3 \mu \mathrm{m}$ aerosol particles in models of the human alveolar sac and terminal acinar bifurcation under rhythmic wall motion for two breathing conditions. Zywczyk and Moskal [34] investigated the fluid flow and the aerosol dynamics in a rhythmically expanding and contracting 2D human alveolus model. Khajeh-Hosseini-Dalasm and Longest [35] reported analytically the flow field dynamics and particle transport on a moving wall model of a pulmonary acinus at six different gravity angles. Another analytical study on a simplified model to predict the alveolar deposition for particle diameters $d_{p} \geq 0.1 \mu \mathrm{m}$ was conducted by Georgakakou et al [36]. More recently, Talaat and Xi [37] examined the effects of various physiological factors including the wall motion modes, particle size, alveolus orientation, breathing frequency, and depth on the flow and particle deposition in expanding-contracting single terminal alveoli. They proposed a correlation for particle deposition in terminal alveoli that captured the separate contributions on the gravitational sedimentation and periodic wall motion. Kolanjiyil and Kleinstreuer [38] stated that particle behavior depends on the lung-airway geometry, particle characteristics, and inhalation flow frequency. Calmet et al [39] investigated the flow features and the micro-particle deposition pattern happening in the human respiratory tract during sniffing. Their analysis showed the flow regime in the nasal cavity to be laminar to transitional. Also in a recent study [40], the researchers simulated airflow and particle accumulation using the Kitaoka airway model and compared them with Weibel's results. They studied how flow rate and the number of generations affect particle accumulation. To analyze the fluid flow and particle transport dynamics in the alveolar region of the lungs, Chakravarty et al [41] used a single alveolar duct model with hemispherical oscillating walls. They reported that the viscous effects largely dominated the flow characteristics in the alveolus.

In summary, the above-mentioned studies indicate that the breathing scenarios and the wall motion are critical factors to the assessment of the airflow and aerosol particle behavior in the pulmonary region of the human lung. However, it can be seen that there are few studies where an ideal human respiratory model is applied for the study of the effects of the alveolar wall motion and the different breathing conditions on airflow, especially for the pulmonary acinus. Determining the airflow in the human pulmonary acinus is always a thorny subject due to its highly complex structure. More studies may help to a better understanding of airflow dynamics and the toxic or therapeutic aerosols transport in this zone. Therefore, it can be more effectively dealt with the systemic lung diseases caused by pathogens such as Covid-19. The focus of this paper is to simulate the unsteady flow field of the human pulmonary acinus. For this purpose, a respiratory bronchiole model, an alveolar duct model, and an alveolar sac model have been utilized for CFD simulations. These $3 \mathrm{D}$ models represent the acinar airways and have the dynamic wall boundaries, i.e., the rhythmically 
expanding/contracting motion of the alveolar wall during respiration. The mouth inlet flow rates of 15,30 , and 60 $\mathrm{L} / \mathrm{min}$ (LPM) for people under light, normal and heavy activities, respectively, have been applied to perform the unsteady respiration.

\section{Materials and method}

The pulmonary region of the lung consists of the respiratory bronchioles (with partly surrounding alveoli), the alveolar ducts (completely covered by alveoli), and the alveolar sacs (the blind-ending terminations of alveolar ducts) [42]. To represent the airflow in these generations, i.e., generation 17 (G17), 18 (G18), and 23 (G23), idealized models developed in the author's previous study [43] have been employed, as shown in figure 1.

The acinar region is not static and stiff structure in that the walls of the alveolar sacs rhythmically expand and contract during each breath. Therefore, there was a minimum distance between the alveoli surrounding the model surfaces to enable the wall movement. The alveolar wall motion during expansion/contraction has a vital role in determining the airflow in the human pulmonary acinus. For an average adult human with a functional residual capacity (FRC) of $3 \mathrm{~L}$ and a total lung capacity of $6 \mathrm{~L}$, it was reported that there was a maximum increase in diameter of an alveoli of 0.26 [38]. Hence, the alveolar wall deformation rate, assuming isotropic volume change spatially, was taken as $25 \%$, except for the rigid-walled alveolar ducts. The respiratory parameters for an average human adult under different breathing conditions were described in detail in the previous study [43]. In this study, the Reynolds numbers at the inlet of airway generations were varied between 0.01 and 1.16 for inhalation flow rates.
Thus, the airflow regime in the pulmonary region was laminar. Thereby, the airflow was described by solving the following 3D governing transport equations for the incompressible flow on a commercial finite-volume based program (Fluent 19.0, ANSYS Inc., Canonsburg, PA).

$$
\nabla \cdot \vec{u}=0
$$

$$
\rho \frac{d \vec{u}}{d t}+\rho(\vec{u} \cdot \nabla) \vec{u}=-\nabla p+\mu \nabla^{2} \vec{u}+\rho \vec{g}
$$

where $\overrightarrow{\mathrm{u}}$ denotes the velocity vector $(\overrightarrow{\mathrm{u}}=[\mathrm{u}, \mathrm{v}, \mathrm{w}]), P$ is the air pressure, $\rho$ is the density, $t$ is time and $\mu$ is the dynamic viscosity. Applying a three-dimensional, pressure-based, and implicit solver with the Least-squares cell-based gradient option for each of the transient breathing condition, the 3D governing equations were solved using Fluent 19.0. In the numerical simulations, the Coupled was selected as a pressure-velocity coupling scheme. On the other hand, the PRESTO discretization scheme and the second-order upwind scheme were used for pressure and momentum, respectively. In the simulations, the fluid properties of the air at $37^{\circ} \mathrm{C}$ were considered.

In this study, the flow rates of 15,30 , and 60 LPM with the same tidal volume $500 \mathrm{~mL}$ were used to simulate the respiration for people under three different activities, i.e., light, normal and heavy activity, respectively. The average velocity at input a given generation is calculated based on the assumption that each airway generation will have two daughter tubes. For example, if the mouth inlet flow rate is $15 \mathrm{LPM}$, the actual flow rate in G17 is $15 / 2^{17} \mathrm{LPM}$, i.e., $11.44 \times 10^{-5}$ LPM. The airflow in G17-G18-G23 was solved for these inspiration flow rates, assuming a parabolic air velocity profile at the model entrance. For the simulations, a uniform gauge pressure condition at the model inlet (atmospheric pressure, $101 \mathrm{kPa}$ ) and the no-

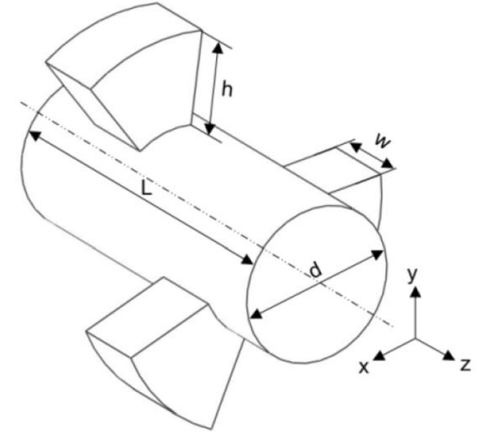

(a)

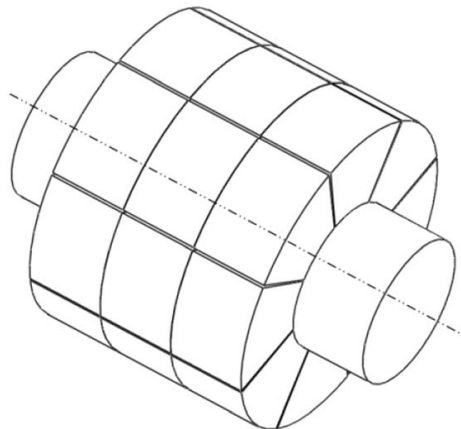

(b)

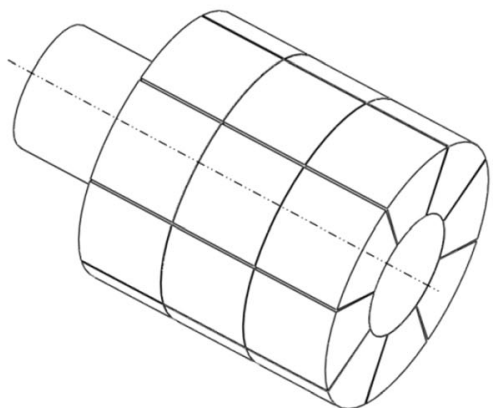

(c)

Figure 1. Three-dimensional models representing the acinar region of human lung; (a) the respiratory bronchiole, (b) the alveolar duct, (c) the alveolar sac. Here, duct length (L): 1200, 920, and $832 \mu \mathrm{m}$; lumen diameter, (d): 500, 470, and $206 \mu \mathrm{m}$; length of alveoli (w): 250 , 250, and $150 \mu \mathrm{m}$; and depth of alveoli (h): 300, 265, and $169 \mu \mathrm{m}$, respectively. 
slip boundary condition on the model walls were applied, assuming gravity in the negative $y$-direction. All the calculations were conducted as double-precision to more accurately determine the airflow behaviour in the acinar region. After a series of sequential breathing cycles, the first inspiration phase was considered to start at time $\mathrm{t}=0 \mathrm{~s}$.

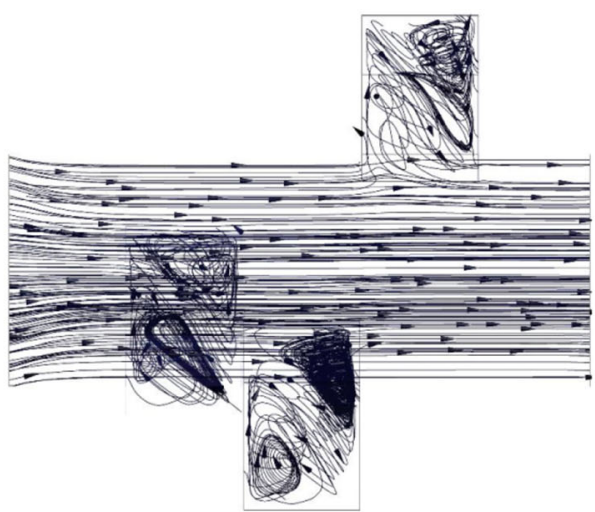

(a)
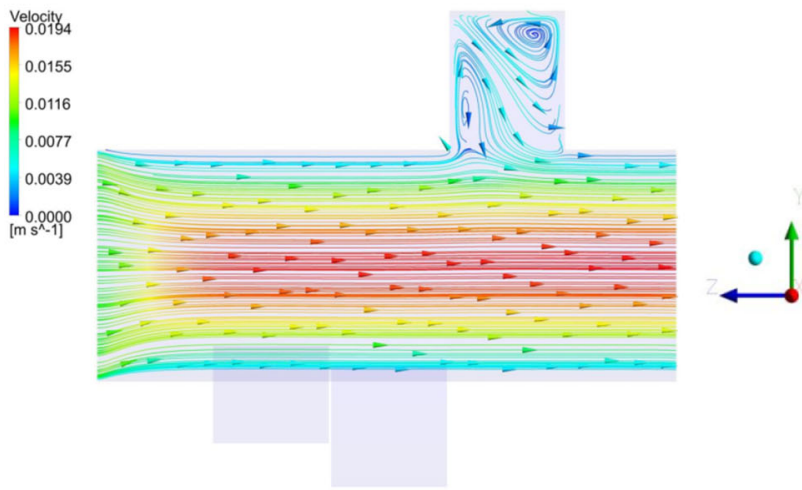

(b)

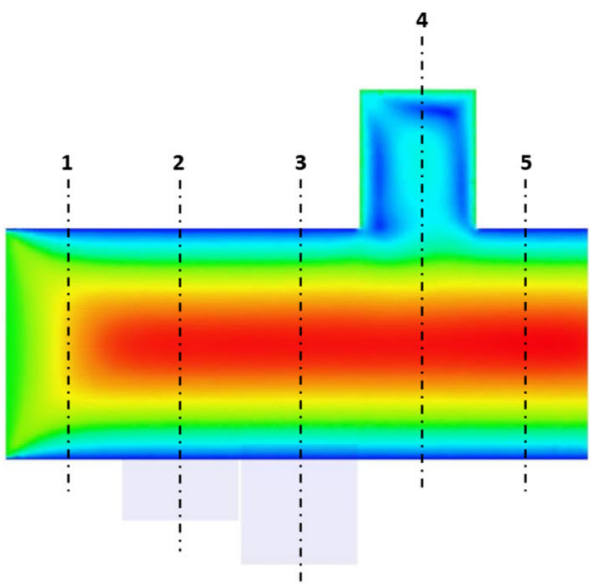

(c)

Figure 2. (a) 3D flow patterns in the respiratory bronchiole model (G17) for the flow rate of 15 LPM ( $R e=0.29)$, (b) $2 \mathrm{D}$ streamline pattern and (c) the contour of velocity magnitude in the yz-plane at the end of inspiration phase.
The computations were carried out for one breathing cycle with $2 \mathrm{~s}$ inspiration immediately followed by $2 \mathrm{~s}$ expiration (without pause). It was assumed that both expansion and contraction followed the square wave inhalation profile. The alveolar wall motion was assumed to be isotropic spatially during respiration in all directions with the volume excursion of $25 \%$. To reduce the numerical uncertainties and to check mesh independence, the preliminary tests for time step and mesh sensitivity were carried out. In order to determine the time step, the average air velocity at the lumen channel centre was taken into account. When the time step was 0.005 , the average air velocity differed only $2 \%$ than that of a time step of $0.05 \mathrm{~s}$. Thus, the time step was fixed at $0.05 \mathrm{~s}$ in all simulations. The transient

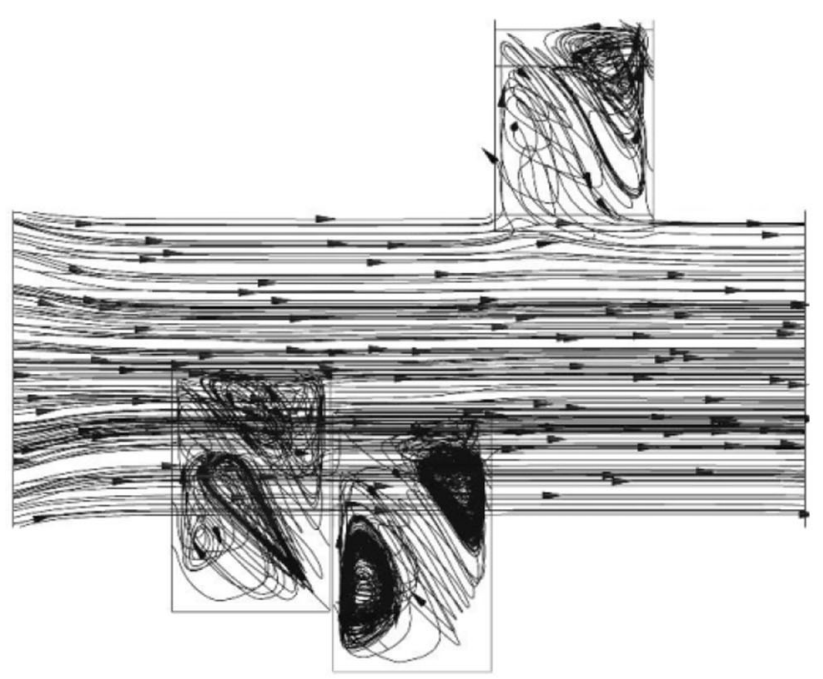

(a)

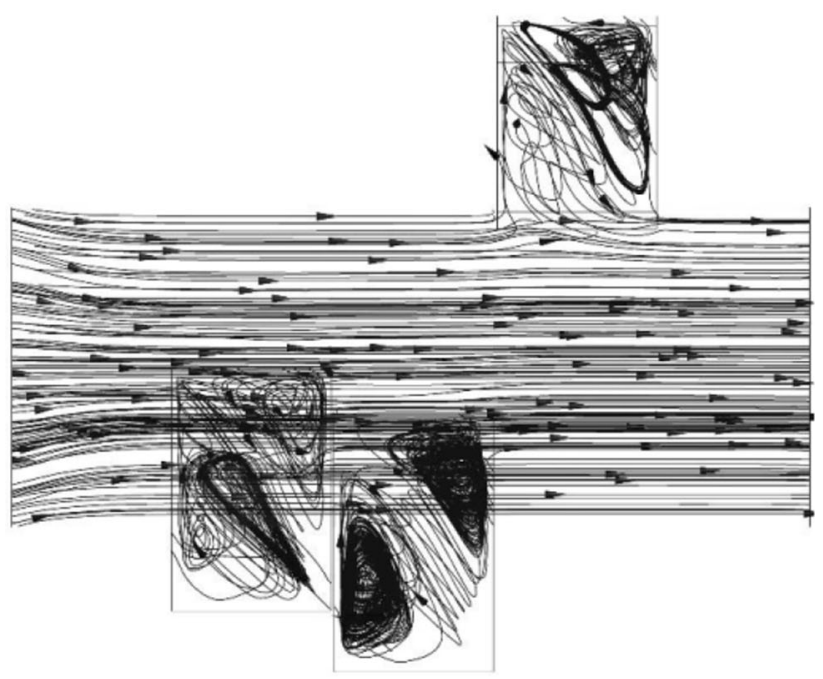

(b)

Figure 3. 3D flow pattern in the respiratory bronchiole model (G17) for the flow rate of (a) $30 \mathrm{LPM}(\mathrm{Re}=0.58)$ and (b) $60 \mathrm{LPM}$ $(\operatorname{Re}=1.16)$ at the end of inspiration. 
simulation for each time step was assumed to converge when the residuals decreased below $10^{-6}$. Additionally, the results for different grid sizes ranging from very coarse to very fine were compared and, therefore, the final generated meshes consisted of 995,248, 2,114,624, and $1,928,570$ tetrahedral elements in all simulations for the respiratory bronchiole, the alveolar duct, and sac model, respectively. Since the element type in the meshing in Fluent 19.0 significantly affects the accuracy of the numerical solution, the quality of the generated mesh

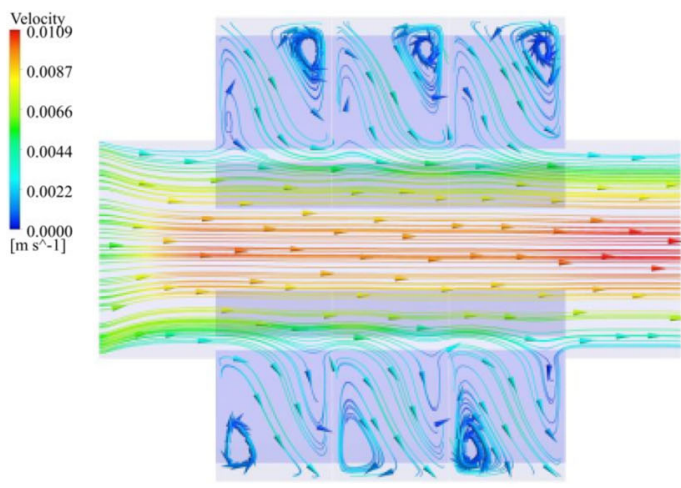

(a)

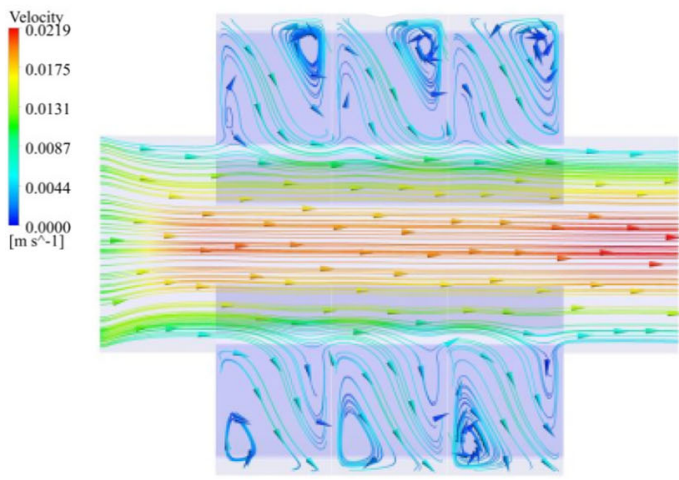

(b)

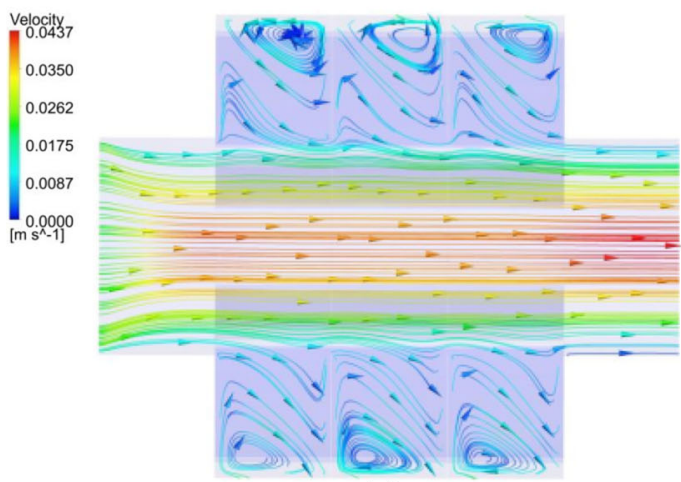

(c)

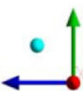

Figure 4. 2D streamline pattern in the alveolar duct model (G18) for the flow rate of (a) $15 \mathrm{LPM}(\mathrm{Re}=0.15)$, (b) $30 \mathrm{LPM}(\mathrm{Re}=0.31)$ and (c) $60 \mathrm{LPM}(\mathrm{Re}=0.62)$ at the end of inspiration. structure was examined in terms of quality using the aspect ratio and skewness criteria of the elements. Aspect ratio is the measure of the stretch of a cell, and its allowable value in Fluent 19.0 is less than 5. The aspect ratio in the current study, for example, was averaged 1.8 for the respiratory bronchiole model. On the other hand, skewness is defined as the difference between the shape of a cell and the shape of an equilateral cell. Here, the value of skewness of the elements was below the 0.85 value suggested by Fluent 19.0. The computations were performed on a DELL workstation with 32 GB RAM and two $2.30 \mathrm{GHz}$ Intel Xeon CPU. The run-time of a transient simulation for one breath cycle lasted from $15 \mathrm{~h}$.

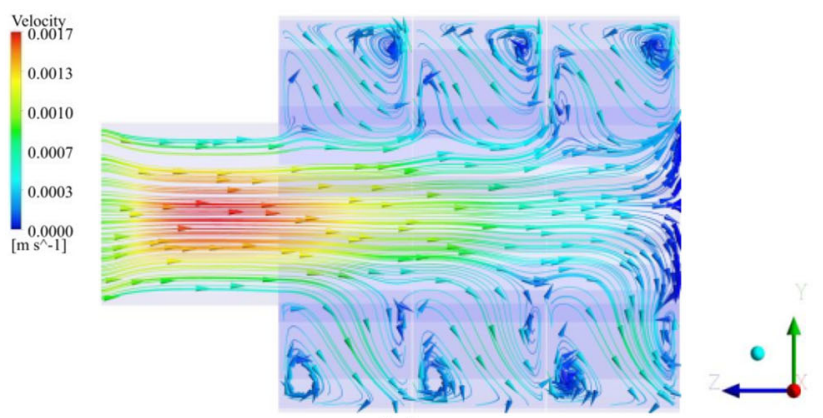

(a)

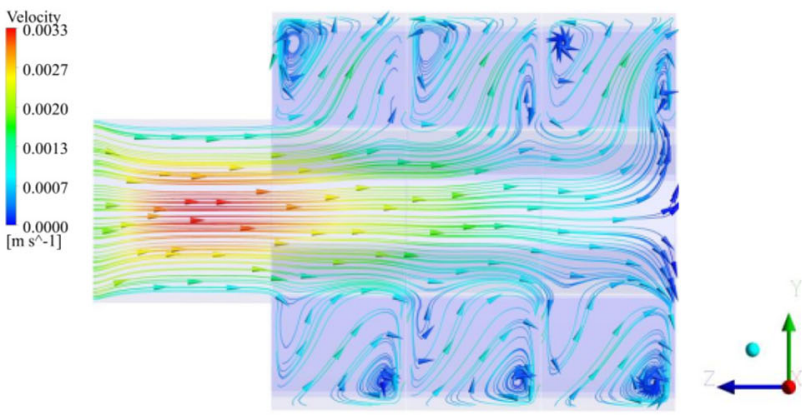

(b)

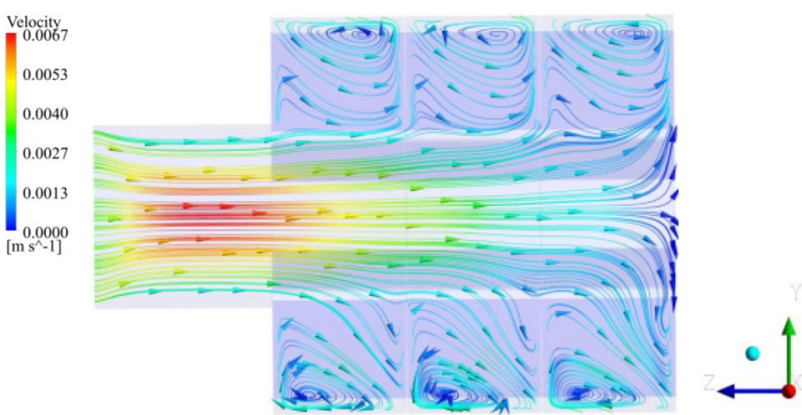

(c)

Figure 5. 2D streamline pattern in the alveolar sac model (G23) for the flow rate of (a) $15 \mathrm{LPM}(\mathrm{Re}=0.01)$, (b) $30 \mathrm{LPM}(\mathrm{Re}=0.02)$ and (c) 60 LPM $(\operatorname{Re}=0.04)$ at the end of inspiration. 


\section{Results and discussions}

The models presenting the human pulmonary acinus are validated with the numerical results of Darquenne et al [28] and Ciloglu et al [43], and, applied for three breathing conditions with mouth flow rates 15 (light), 30 (normal), and 60 (heavy) LPM for an average human adult with tidal volume $500 \mathrm{~mL}, \mathrm{FRC}$ of $3 \mathrm{~L}$ and total lung capacity $6 \mathrm{~L}$. During one breathing cycle, equal inspiration and expiration times are assumed.

\subsection{Flow in the respiratory bronchial during inspiration}

Figures 2 and 3 depict color-coded 3D flow patterns as well as the streamlines and contours of velocity magnitude onto yz-plane in the respiratory bronchial model (G17) at the end of inspiration $(t=2 s)$ for three breathing conditions. As seen in figures $2(a-b)$ and $3(a-b)$, in most of the central lumen channel, the streamlines are parallel to the longitudinal pathway. The streamlines formed a curvilinear shape near the alveoli mouths. Indeed, the streamlines appear to be divided into two regions due to the rhythmic expansion/contraction rate of alveoli and the pressure gradients in the outer flow. One is the alveolar flow and the other is towards the lumen channel as bulk flow (see figure 2 (b)). Moreover, the alveolar cavities disrupt the external smooth flow during inspiration. The net effects of unsteady channel flow and the alveolar wall motion cause the recirculation of a small amount of air entering the alveoli, as observed in figure 2 ( $a$ and $b$ ). The recirculation eddies were caused by the sudden entry of fluid into the alveolus and determined the alveolar flow characteristic [44]. As seen in figure 2 (c), the velocities in the recirculation regions are slower when compared to channel flow, consistent with the data of previous studies $[26,45,46]$. On the other hand, the flow velocity in the central alveolar cavity is greater than that of the near of alveolar wall (see figure 2 (c)).

\subsection{Flow in the alveolar duct and the alveolar sac during inspiration}

Figures 4 and 5 show the 2D streamlines onto the yz-plane for lumen channel and alveoli that are placed symmetrically on two opposite sides of the air-carrying lumen channel in the alveolar duct (G18) and alveolar sac (G23) model, respectively. Although the flow patterns are similar to each other at low Reynolds numbers, the differences in the streamlines were observed as the Reynolds number increased (figure 4 (c)). This can be attributed to the fact that the flow structure changes only negligibly with the Reynolds number as long as it remains small [47]. In these figures, it is also observed that the center of eddies has appeared in the distal regions of alveolar cavities, namely the downstream zones. It is shown that the intensity of the recirculating flow is dependent on the Reynolds number (or breathing scenario) and it increases with increasing the Reynolds number. As one recirculation zone grows the other becomes smaller, and these asymmetrical flow patterns increase with increasing the Reynolds number. It should be noted that the researchers also investigated the flow in the channels with a one-third extension and a sudden expansion, and they found that the flow structure depended on numerical and physical parameters as well as a numerical technique [48, 49]. However, although the alveoli dimensions and Reynolds numbers are the same, it is observed that there are differences in the intensity of the recirculating flow. This is attributed to the presence of a different form of entrainment region in each alveoli mouth [29]. In another study, Zhang and Kleinstreuer [50] simulated the 3D airflow structures and micron-particle transport in the triple bifurcations under normal breathing and highfrequency ventilation conditions. They also observed the flow asymmetries caused by differences between flow acceleration and deceleration during normal breathing, especially for inspiratory flow. As the generation number increases (see figure 5 (b)), the flow pattern in the alveoli turns into the radial flow while the recirculating flow intensity is decreasing. As can be seen in figures 5 (a) and (b), a large part of the channel flow is directed into the alveolar cavities due to the blind-ended termination of the alveolar canals, resulting in increased mass transfer from the lumen channel to alveoli.

\subsection{Flow in the alveolar cavities}

Considering its structure, an alveolus acts like a stenosed channel, i.e., a suddenly expanding/contracting channel. The stenotic flows with the low-Reynolds-number have been investigated by researchers using three-dimensional stenosis [51], an axisymmetric expanding pipe [52], an airway model [53] and the stenosed tubes [54]. Studies showed that the stenotic flow exhibited very different characteristics such as the flow instabilities, the perturbations, the recirculated flow, the secondary flow motions, or the separated flow regions, resulting in the periodic flow transitions in the stenotic regions. In this study, the rhythmical expansion and contraction of the alveolar walls (the alveolar wall motion) induced the alveolar airflow. The alveolar flow is a low-Reynolds-number periodic flow event that occurs in open cavities [30]. Alveolar cavities are generally relatively stagnant pockets that include somehow varying flow conditions with temporary perturbations and exhibit weak transport properties. In the present study, the recirculation eddies emerged from the mouth of the alveoli in the transient flow conditions, and, their centres shifted towards the distal regions of the alveoli with the increase of the Reynolds numbers (figure 4 (b) and 5 (b)). On the other hand, in such a stenoic channel, the flow velocities are 


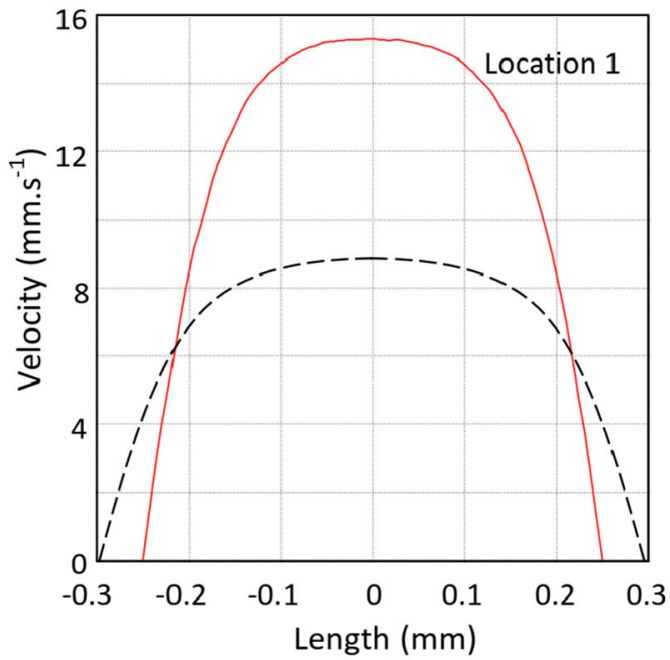

Present model prediction

-- - Experimental observation by

Ertbrugen et al. [27]

(a)

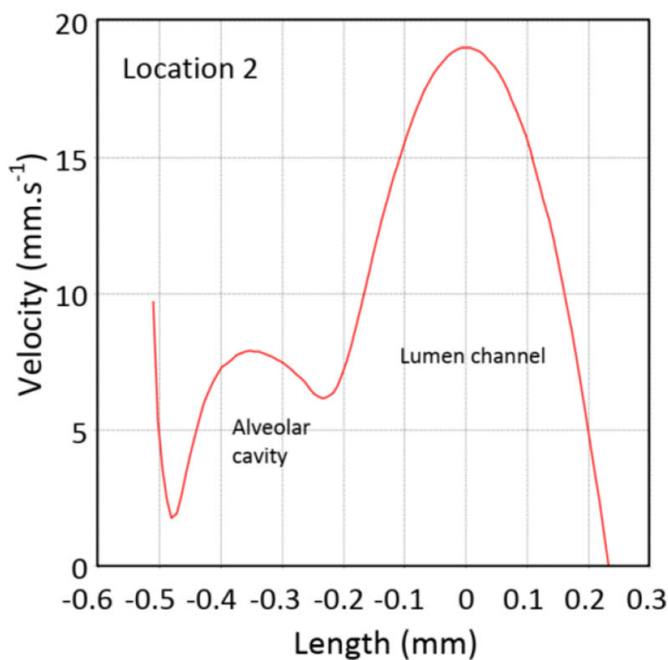

(b)

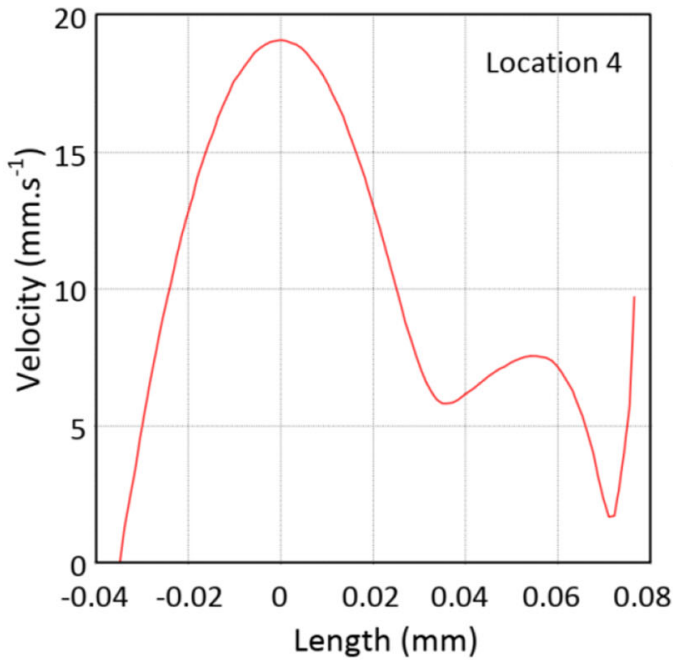

(d)

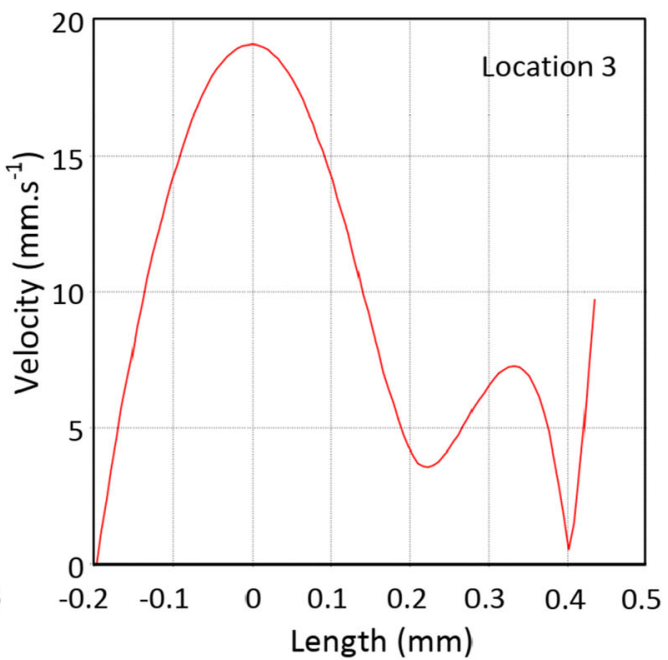

(c)

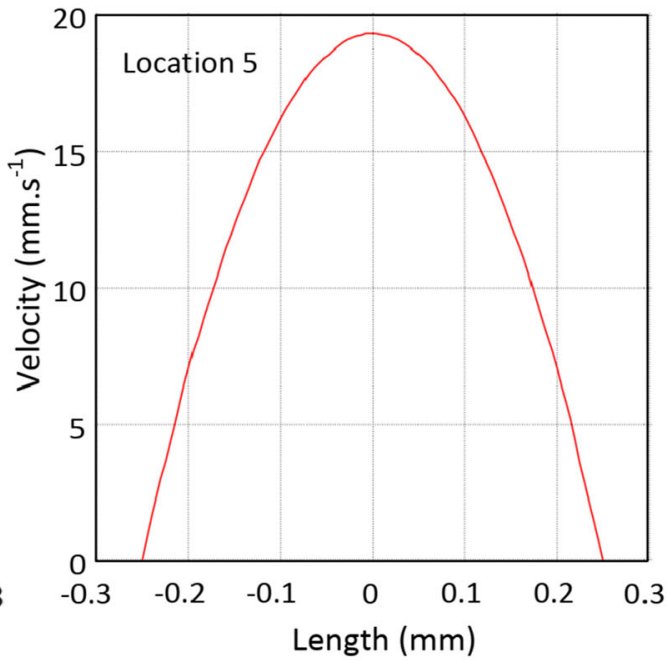

(e)

Figure 6. The velocity profiles along with different locations of the respiratory bronchial model (G17). Note that Ertbruggen's experimental results [27] were obtained for G21 with $0.3 \mathrm{~mm}$ in lumen diameter and the maximum velocity was $8.92 \mathrm{~mm} . \mathrm{s}^{-1}$. Both the current study and the experimental study reported a parabolic velocity profile in the acinar region. 

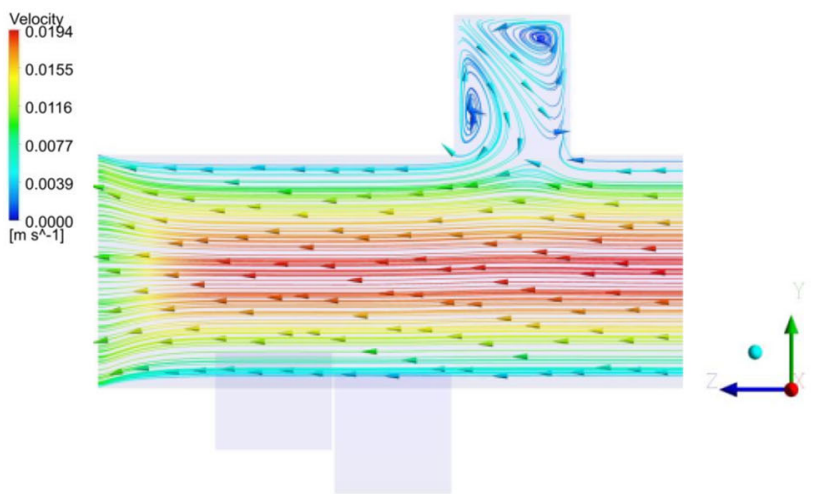

(a)

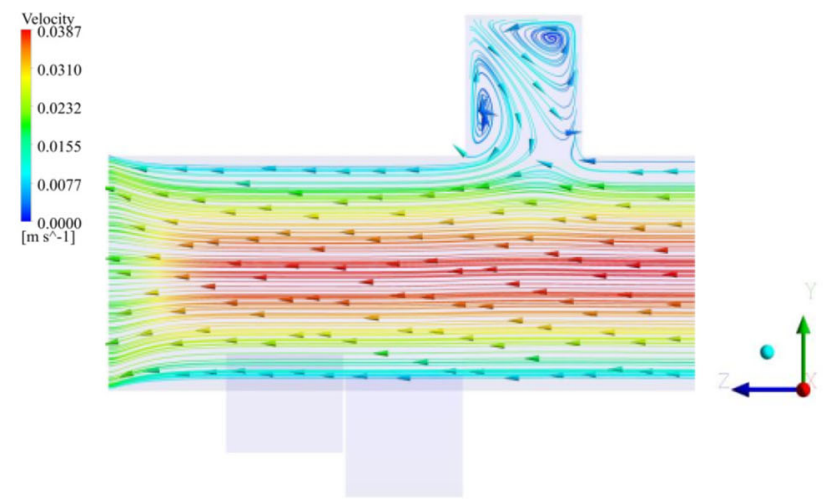

(b)

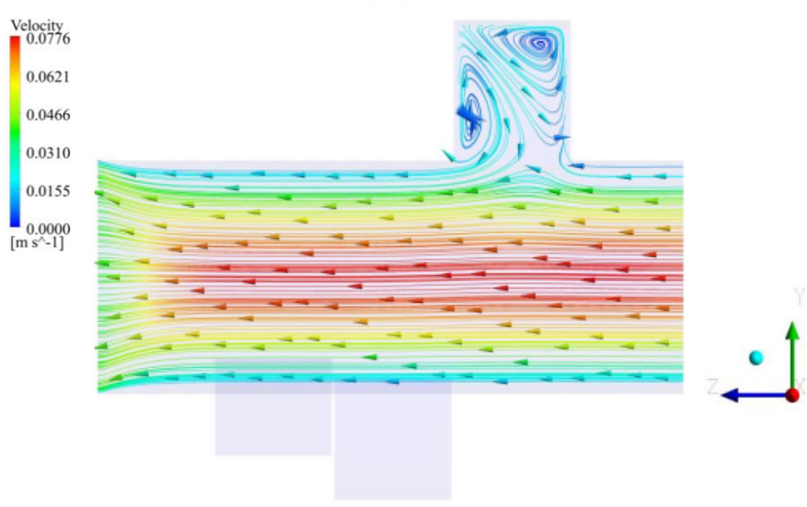

(c)

Figure 7. 2D streamline pattern in $\mathrm{G} 17$ for the flow rate of (a) 15 LPM, (b) 30 LPM and (c) 60 LPM at the end of expiration.

lower than that of the channel flow (figure 2 (c)). This situation was similar to the flow characteristics in a stenoic blood vessel [44]. The sudden contraction in flow geometry creates a flow barrier, causing a decrease in the flow velocity $[44,54]$. As mentioned before, in this study, it has been observed that flow irreversibilities and eddies occur in the alveolar flow regions. This is because of the instability of the flow field induced by the stenoic channel. Besides, these instabilities have been also attributed to the nonuniform static properties of the alveolar fluid lining, nonuniform deformation of the lung tissue, and avalanche

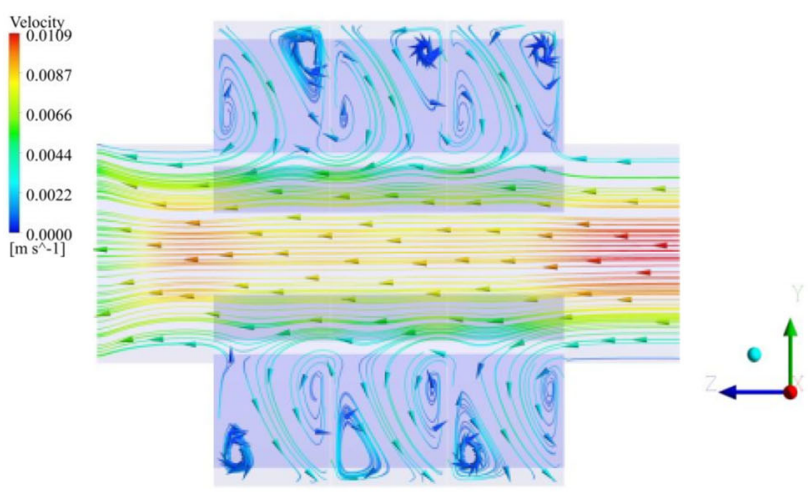

(a)

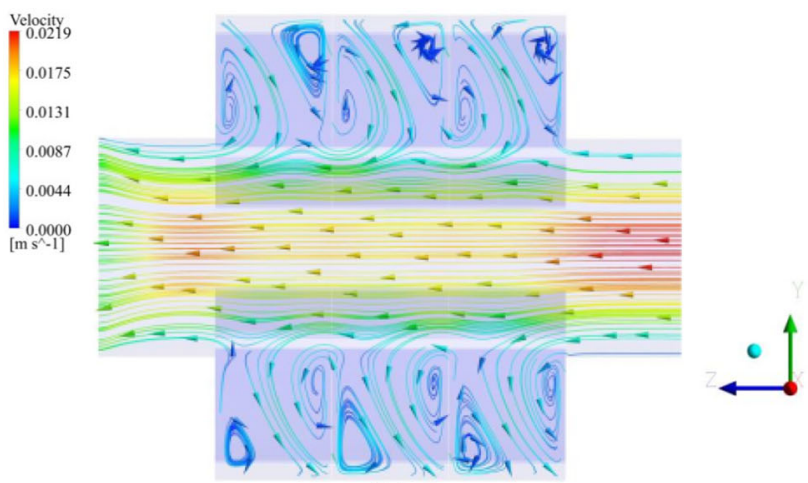

(b)

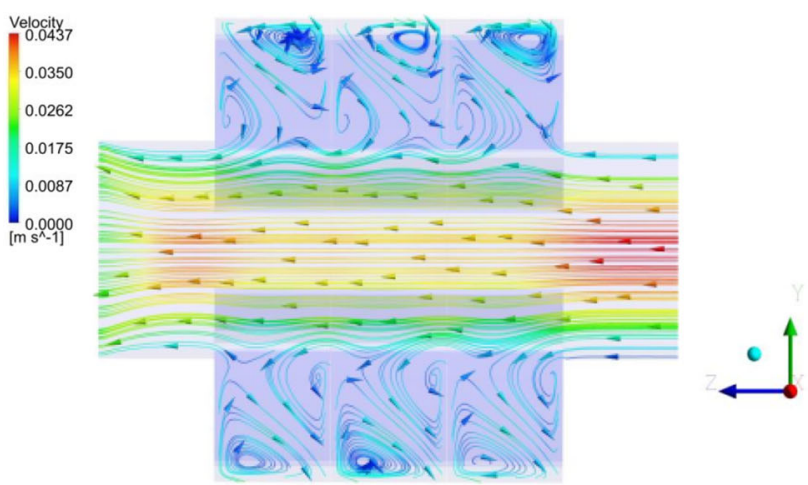

(c)

Figure 8. 2D streamline pattern in G18 for the flow rate of (a) 15 LPM, (b) 30 LPM and (c) 60 LPM at the end of expiration.

shocks resulting from the sudden swelling and discharge of many alveoli due to the transient flow [55-58]. It can be concluded that the airflow behavior in the human pulmonary acinus is strongly dependent on the low-Reynoldsnumber flow conditions.

\subsection{The velocity profiles along different sections of the respiratory bronchial model}

In figure 6 , the velocity profiles obtained by this study are presented at five locations on the respiratory bronchial 


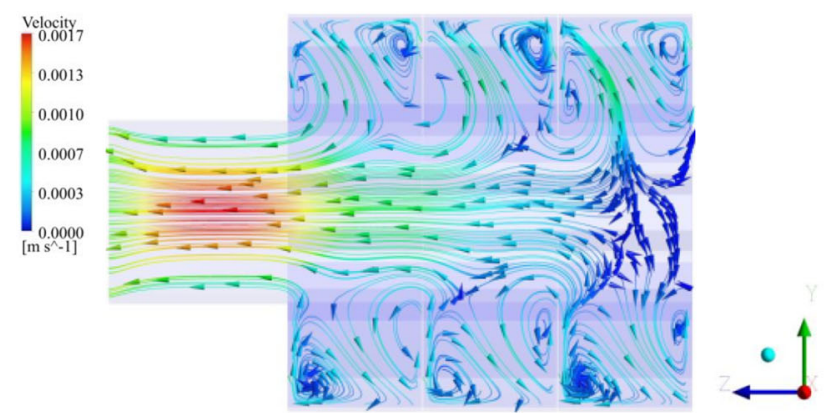

(a)

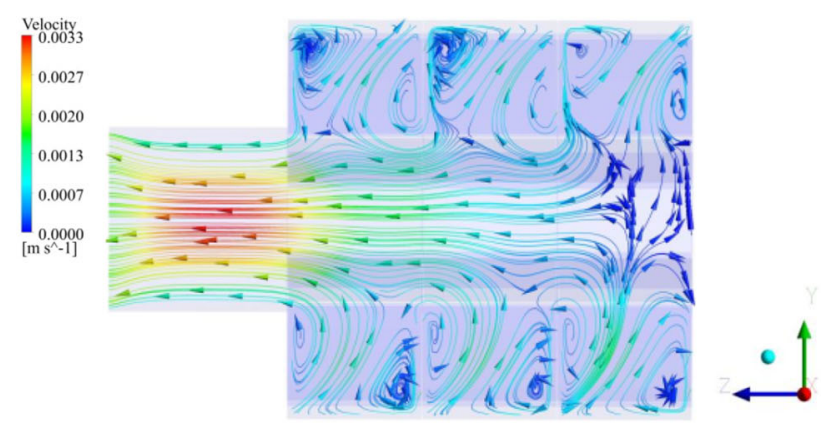

(b)

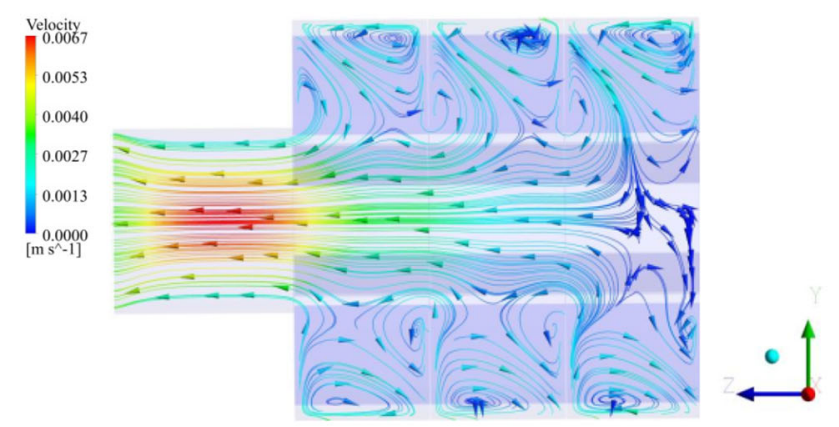

(c)

Figure 9. 2D streamline pattern in G23 for the flow rate of (a) 15 LPM, (b) 30 LPM and (c) 60 LPM at the end of expiration.

model for the flow rate of 15 LPM (in light activity). These locations can be shown in figure 2 (c). As shown in figures 6 (a) and (e), the velocity profiles in the lumen channel have a parabolic shape and conform to the Ertbruggen's experimental results [27] obtained for generation 21. In locations 2, 3, and 4, when approaching the alveolar cavities, the flow pattern is disrupted due to the recirculating airflow. As mentioned above, the flow slows down when entering the alveoli. Indeed, the velocity magnitudes inside the cavities are smaller than twice that of the lumen channel, as seen in figure 6 (b). Since the distance required for the fully developed flow (i.e., less than $0.1 \mathrm{~mm}$ ) is included in this model, the velocity profile has regained its parabolic shape. On the other hand, for light breathing conditions the maximum velocity predicted in the simulation is $19.33 \mathrm{~mm} . \mathrm{s}^{-1}$ and occurs in location 5 (see figure 6 (e)).

\subsection{Flow characteristics during expiration}

Figures 7, 8, and 9 illustrate the flow characteristics in the present models at the end of the expiration phase $(t=4 \mathrm{~s})$. Essentially, the inhalation is the intake of inhaled air into the lungs until the internal lung pressure is equal to the atmospheric pressure. The exhalation is the return of highpressure-air in the alveolated ducts to the atmosphere as the chest muscles relax to their normal positions. In this case, the flow direction and the alveolar wall movement is reversed, causing the model to contraction. Thus, the flow recirculates again in the central region within the alveolus and then heads towards the lumen channel from the alveolar cavity, in contrast to that of the inspiration phase. In this distal lung zone, the Reynolds number representing the flow rate of 15 LPM is very low, bringing about unsteady flow characteristics. Likewise the inspiration phase, the instability phenomena were observed in the exhalation phase. In figure 7, the similar flow patterns were observed for all flow situations. In the deeper region of the lung, however, the varieties in the streamlines were appeared, depending on the breathing scenarios (see figures 8-9). The simulations also showed the presence of transient recirculation eddies. This transient flow recycles may have contributed to the weak flow irreversibility observed in the present study. Some researchers have also reported similar flow instabilities in their experimental and numerical studies for Taylor-Coutte flow [59], pipe jet flow [60], and flow in two-dimensional jets [61]. As a consequence, the airflow in the pulmonary region is influenced by the intensity of the recirculating flow and eddies inside the alveoli [29, 62, 63].

\section{Conclusions}

The aim of this work was to numerically investigate the behavior of airflow in the human pulmonary acinus during transient inspiration. The simulations were performed on three simplified models for three breathing scenarios such as light (15 LPM), normal (30 LPM), and heavy breathing conditions (60 LPM). It was determined that the airflow in the pulmonary zone was characterized by the recirculation regions in the alveolar cavities. Compared to the channel flow, the velocities in the alveoli were slower. The fluid transfer to the alveoli was the result of the interaction between the unsteady channel flow and the alveolar wall motion. For all breathing conditions, the recirculation regions were observed. The intensity of the recirculating flow increased with the increase in Reynolds number. The change in the recirculation intensity in the alveoli can be an important parameter in determining the airflow and particle behaviour in the pulmonary acinus. In fact, this region contains densely branching air channels. Therefore, it is clear that considering the cyclic alveolar wall motion and the different breathing conditions in the model will bring a 
new perspective to the simulation of acinar airflow. In addition, this analysis of the flow phenomenon discussed here provides a better understanding of the ventilation in the deep lung regions. In the future, if relevant models are established and strong consensus develops, it can contribute to the development of effective treatment methods for patients struggling with systemic lung diseases. It can also find application in medical conditions where gas exchange breaks down by pathogens such as Covid-19, i.e., postCovid fibrosis. Consequently, to simulate or estimate the airflow dynamics and even for local deposition of toxic/ therapeutic aerosols, the results obtained in this study can be easily utilized into the human lung airway models.

$\begin{array}{ll}\text { Abbreviations } \\ \text { ARDS } & \text { Acute respiratory distress syndrome } \\ \text { CFD } & \text { Computational fluids dynamics } \\ \text { FRC } & \text { Functional residual capacity } \\ \text { LPM } & \text { Liter per minute } \\ \text { d } & \text { Lumen diameter } \\ \text { G } & \text { Generation number } \\ \text { g } & \text { Gravity } \\ \text { h } & \text { Depth of alveoli } \\ \text { L } & \text { Duct length } \\ \text { p } & \text { Pressure } \\ \text { t } & \text { Time } \\ \text { u } & \text { Fluid velocity } \\ \text { w } & \text { Length of alveoli } \\ \mu & \text { Dynamic viscosity } \\ \rho & \text { Density }\end{array}$

\section{References}

[1] Arabi Y M, Mandourah Y, Al-Hameed F, Sindi A A, Almekhlafi G A, Hussein M A, Jose J, Pinto R, Al-Omari A, Kharaba A, Almotairi A, Al Khatib K, Alraddadi B, Shalhoub S, Abdulmomen A, Ismael Qushmaq, Mady A, Solaiman O, Al-Aithan A M, Al-Raddadi R, Ragab A, Balkhy H H, Al Harthy A, Deeb A M, Al Mutairi H, AlDawood A, Merson L, Hayden F G and Fowler R A 2018 Corticosteroid therapy for critically III patients with Middle East respiratory syndrome. Am. J. Respir. Crit. Care Med. 197: 757-767

[2] Lee N, Allen Chan K C, Hui D S, Ng E K O, Wu A, Chiu R W K, Wong V W S, Chan P K S, Wong K T, Wong E, Cockram C S, Tam J S, Sung J J Y and Lo Y M D 2004 Effects of early corticosteroid treatment on plasma SARSassociated Coronavirus RNA concentrations in adult patients. J. Clin. Virol. 31: 304-309

[3] Barut F, Ozacmak V H, Turan I, Sayan-Ozacmak H and Aktunc E 2016 Reduction of acute lung injury by administration of spironolactone after intestinal ischemia and reperfusion in rats. Clin. Investig. Med. Med. 39: 15-24
[4] Yavas G, Yavas C, Celik E, Sen E, Ata O and Afsar R E 2019 The impact of spironolactone on the lung injury induced by concomitant trastuzumab and thoracic radiotherapy. Int. J. Radiat. Res. 17: 87-95

[5] Zannad F, Alla F, Dousset B, Perez A and Pitt B 2000 Limitation of excessive extracellular matrix turnover may contribute to survival benefit of spironolactone therapy in patients with congestive heart failure: insights from the randomized aldactone evaluation study (RALES). Rales Investigators Circulation. 102: 2700-2706

[6] Ware L B 2006 Pathophysiology of acute lung injury and the acute respiratory distress syndrome. Semin. Respir. Crit. Care Med. 27: 337-349

[7] MacLaren R and Stringer K A 2007 Emerging role of anticoagulants and fibrinolytics in the treatment of acute respiratory distress syndrome. Pharmacotherapy. 27: 860-873

[8] Ware L B, Camerer E, Welty-Wolf K, Schultz M J and Matthay M A 2006 Bench to bedside: targeting coagulation and fibrinolysis in acute lung injury. Am. J. Physiology Lung Cell Mol. Physiol. 291: 307-311

[9] Hardaway R M, Harke H, Tyroch A H, Williams C H, Vazquez Y and Krause G F 2001 Treatment of severe acute respiratory distress syndrome: a final report on a phase I study. Am. Surg. 67: 377-382

[10] Biot C, Daher W, Chavain N, Fandeur T, Khalife J, Dive D and Clercq E D 2006 Design and synthesis of hydroxyferroquine derivatives with antimalarial and antiviral activities. J. Med. Chem. 49: 2845-2849

[11] Grein J, Ohmagari N, Shin D, Diaz G, Asperges E, Castagna A, Feldt T, Green G, Green https://pubmed.ncbi.nlm.nih. gov/32275812/-affiliation-1 M L, Lescure F, Nicastri E, Oda R, Yo K, Quiros-Roldan E, Studemeister A, Redinski J, Ahmed S, Bernett https://pubmed.ncbi.nlm.nih.gov/ 32275812/-affiliation-1 J, Chelliah https://pubmed.ncbi.nlm. nih.gov/32275812/-affiliation-1 D, Chen D, Chihara S, Cohen S H, Cunningham J, Monforte A D, Ismail S, Kato H, Lapadula G, L'Her E, Maeno T, Majumder S, Massari M, Mora-Rillo M, Mutoh Y, Nguyen D, Verweij E, Zoufaly A, Osinusi A O, DeZure A, Zhao Y, Zhong L, Chokkalingam A, Elboudwarej E, Telep L, Timbs L, Henne I, Sellers S, Cao H, Tan S K, Winterbourne https://pubmed. ncbi.nlm.nih.gov/32275812/-affiliation-1 L, Desai P, Mera R, Gaggar A, Myers R P, Brainard D M, Childs R and Flanigan T 2020 Compassionate use of remdesivir for patients with severe Covid-19. N. Engl. J. Med. 382: 2327-2336

[12] Lu C C, Chen M Y, Lee W S and Chang Y L 2020 Potential therapeutic agents against COVID-19: What we know so far. J. Chin. Med. Assoc. 83: 534-536

[13] Caly L, Druce J D, Catton M G, Jans D A and Wagstaff K M 2020 The FDA-approved drug ivermectin inhibits the replication of SARS-CoV-2 in vitro. Antivir Res. 178: 104787

[14] Weibel E R 1963 Morphometry of the Human Lung. Springer, Heidelberg

[15] Dailey H L and Ghadiali S N 2007 Fluid-structure analysis of microparticle transport in deformable pulmonary alveoli. Aerosol Science. 38: 269-288

[16] Cheng K H, Cheng Y S, Yeh H C, Guilmette R A, Simpson S Q, Yang Y H and Swift D L 1996 In vivo measurements of nasal airway dimensions and ultrafine aerosol deposition in 
the human nasal and oral airways. J. Aerosol Sci. 27: 785-801

[17] Cryan S A, Sivadas N and Garcia-Contreras L 2007 In vivo animal models for drug delivery across the lung mucosal barrier. Adv. Drug Deliv. Rev. 59: 1133-1151

[18] Newman S P, Pitcairn G R, Hirst P H and Rankin L 2003 Radionuclide imaging technologies and their use in evaluating asthma drug deposition in the lungs. Adv. Drug Deliv. Rev. 55: 851-867

[19] Jedelsky J, Lizal F and Jicha M 2012 Characteristics of turbulent particle transport in human airways under steady and cyclic flows. Int. J. Heat Fluid Flow. 35: 84-92

[20] Longest P W and Xi J 2007 Effectiveness of direct Lagrangian tracking models for simulating nanoparticle deposition in the upper airways. Aerosol Sci. Technol. 41: 380-397

[21] Li Z, Kleinstreuer C and Zhang Z 2007 Simulation of airflow fields and microparticle deposition in realistic human lung airway models. Part II: particle transport and deposition. Eur. J. Mech. B Fluids. 26: 650-668

[22] Banko A J, Coletti F, Elkins C J and Eaton J K 2016 Oscillatory flow in the human airways from the mouth through several bronchial generations. Int. J. Heat Fluid Flow. 61: 45-57

[23] Kim J, Xi J, Si X, Berlinski A and Su W C 2014 Hood nebulization: effects of head direction and breathing mode on particle inhalability and deposition in a 7-month-old infant model. J. Aerosol Med. Pulm. D. 27: 209-218

[24] Hofmann W, Balásházy I and Koblinger L 1995 The effect of gravity on particle deposition patterns in bronchial airway bifurcations. J. Aerosol Sci. 26: 1161-1168

[25] Darquenne C 2001 A realistic two-dimensional model of aerosol transport and deposition in the alveolar zone of the human lung. J. Aerosol Sci. 32: 1161-1174

[26] Harrington L, Kim Prisk G and Darquenne C 2006 Importance of the bifurcation zone and branch orientation in simulated aerosol deposition in the alveolar zone of the human lung. J. Aerosol Sci. 37: 37-62

[27] Ertbruggen C, Corieri P, Theunissen R, Riethmuller M L and Darquenne C 2008 Validation of CFD predictions of flow in a 3D alveolated bend with experimental data. J. Biomech. 41: 399-405

[28] Darquenne C, Harrington L and Prisk G K 2009 Alveolar duct expansion greatly enhances aerosol deposition: a threedimensional computational fluid dynamics study. Phil. Trans. R. Soc. A. 367: 2333-2346

[29] Kumar H, Tawhai M H, Hoffman E A and Lin C L 2009 The effects of geometry on airflow in the acinar region of the human lung. J. Biomech. 42: 1635-1642

[30] Kumar H, Tawhai M H, Hoffman E A and Lin C L 2011 Steady streaming: a key mixing mechanism in lowReynolds-number acinar flows. Phys. Fluids. 23: 041902

[31] Salma I, Balashazy I, Hofmann W and Zaray G 2002 Effect of physical exertion on the deposition of urban aerosols in the human respiratory system. J. Aerosol Sci. 33: 983-997

[32] Kolanjiyil A V and Kleinstreuer C 2016 Computationally efficient analysis of particle transport and deposition in a human whole-lung-airway model. Part I: theory and model validation. Comput. Biol. Med. 79: 193-204

[33] Ma B and Darquenne C 2011 Aerosol deposition characteristics in distal acinar airways under cyclic breathing conditions. J. Appl. Physiol. 110: 1271-1282
[34] Żywczyk $€$ and Moskal A 2012 Modeling of the influence of tissue mechanical properties on the process of aerosol particles deposition in a model of human alveolus. J. Drug Del. Sci. Tech. 22: 153-159

[35] Khajeh-Hosseini-Dalasm N and Longest P W 2015 Deposition of particles in the alveolar airways: Inhalation and breath-hold with pharmaceutical aerosols. J. Aerosol Sci. 79: $15-30$

[36] Georgakakou S, Gourgoulianis K, Daniil Z and Bontozoglou V 2016 Prediction of particle deposition in the lungs based on simple modeling of alveolar mixing. Respir. Physiol. Neurobiol. 225: 8-18

[37] Talaat K and Xi J 2017 Computational modeling of aerosol transport, dispersion, and deposition in rhythmically expanding and contracting terminal alveoli. J. Aerosol Sci. 112: 19-33

[38] Kolanjiyil A V and Kleinstreuer C 2017 Computational analysis of aerosol-dynamics in a human whole-lung airway model. J. Aerosol Sci. 114: 301-316

[39] Calmet H, Houzeaux G, Vázquez M, Eguzkitza B, Gambaruto A M, Bates A J and Doorly D J 2018 Flow features and micro-particle deposition in a human respiratory system during sniffing. J. Aerosol Sci. 123: 171-184

[40] Kim Y H, Tong Z B, Chan H K and Yang R Y 2019 CFD modelling of air and particle flows in different airway models. J. Aerosol Sci. 134: 14-28

[41] Chakravarty A, Patankar N A and Panchagnula M V 2019 Aerosol transport in a breathing alveolus. Phys. Fluids. 31: 121901

[42] Haefeli-Bleuer B and Weibel E R 1988 Morphometry of the human pulmonary acinus. The Anatomical Record. 220: 401-414

[43] Ciloglu D 2020 A numerical study of the aerosol behavior in intra-acinar region of a human lung. Physics of Fluids. 32: 103305

[44] Berger S A and Jou L D 2000 Flows in stenotic vessels. Annu. Rev. Fluid Mech. 32: 347-382

[45] Darquenne C and Paiva M 1996 Two- and three-dimensional simulations of aerosol transport and deposition in alveolar zone of human lung. J. Appl. Physiol. 80: 1401-1414

[46] Sznitman J, Heimsch F, Heimsch T, Rusch D and Rösgen T 2007 Threedimensional convective alveolar flow induced by rhythmic breathing motion of the pulmonary acinus. $J$. Biomech. Eng. 129: 658-665

[47] Lee D Y and Lee J W 2003 Characteristics of particle transport in an expanding or contracting alveolated tube. $J$. Aerosol Sci. 34: 1193-1215

[48] Sobey I J and Mullin T 1993 Calculation of multiple solutions for the dimensional Navier-Stokes equations. Proceedings of Numerical Methods in Fluid Dynamics, edited by M J Baines and K W Morton (Oxford University Press). Oxford. 4: 417

[49] Drikakis D 1997 Bifurcation phenomena in incompressible sudden expansion flows. Phys. Fluids 9: 76-87

[50] Zhang Z and Kleinstreuer C 2002 Transient airflow structures and particle transport in a sequentially branching lung airway model. Phys. Fluids. 14: 862-880

[51] Mallinger F and Drikakis D 2002 Instability in threedimensional, unsteady, stenotic flows. Int. J. Heat Fluid Flow. 23: 657-663 
[52] Cantwell C D, Barkley D and Blackburn H M 2010 Transient growth analysis of flow through a sudden expansion in a circular pipe. Phys. Fluids. 22: 034101

[53] Choi J, Tawhai M H, Hoffman E A and Lin C L 2009 On intra- and intersubject variabilities of airflow in the human lungs. Phys. Fluids. 21: 101901

[54] Varghese S S, Frankel S H and Fischer P F 2007 Direct numerical simulation of stenotic flows. Part 1. Steady flow. $J$. Fluid Mech. 582: 253-280

[55] Alencar A M, Arold S P, Buldyrev S V, Majumdar A, Stamenović D, Stanley H E and Suki B 2002 Dynamic instabilities in the inflating lung. Nature. 417: 809-811

[56] Fung Y C 1975 Does the Surface Tension Make the Lung Inherently Unstable? Circ. Res. 37: 497-502

[57] McIlwaine M, Bradley J, Elborn J S and Moran F 2017 Personalising airway clearance in chronic lung disease. Eur. Respir. Rev. 26: 160086
[58] Mead J, Turner J M, Macklem P T and Little J B 1967 Significance of the relationship between lung recoil and maximum expiratory flow. J. Appl. Physiol. 22: 95-108

[59] Pfister G, Schmidt H, Cliffe K A and Mullin T 1988 Bifurcation phenomena in Taylor-Couette flow in a very short annulus. J. Fluid Mech. 191: 1

[60] Iribarne A, Frantisak F, Hummel R L and Smith J H 1972 An experimental study of instabilities and other flow properties of a laminar pipe jet. AIChE J. 18: 689

[61] Sato H 1959 The stability and transition of a twodimensional jet. J. Fluid Mech. 7: 53

[62] Kolanjiyil A V and Kleinstreuer C 2019 Modeling Airflow and Particle Deposition in a Human Acinar Region. Comput. Math. Methods Med. 5952941

[63] Haghnegahdar A, Zhao J and Feng Y 2019 Lung aerosol dynamics of airborne influenza A virus-laden droplets and the resultant immune system responses: an in silico study. $J$. Aerosol. Sci. 134: 34-55 